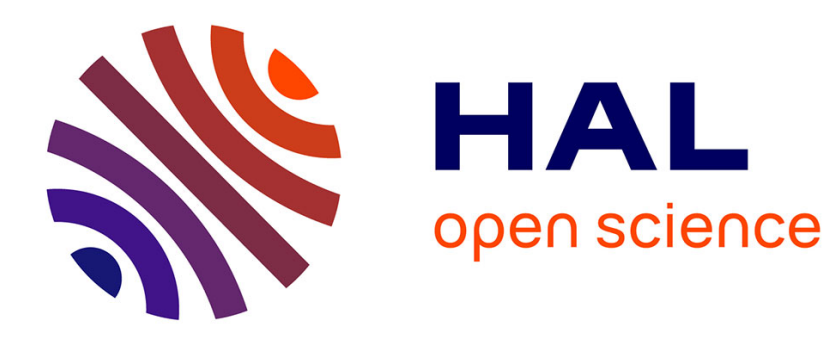

\title{
Generalized Affine Functions and Generalized Differentials
}

Thi Hong Linh Nguyen, Jean-Paul Penot

\section{To cite this version:}

Thi Hong Linh Nguyen, Jean-Paul Penot. Generalized Affine Functions and Generalized Differentials. 2006. hal-00423370

\author{
HAL Id: hal-00423370 \\ https://hal.science/hal-00423370
}

Preprint submitted on 9 Oct 2009

HAL is a multi-disciplinary open access archive for the deposit and dissemination of scientific research documents, whether they are published or not. The documents may come from teaching and research institutions in France or abroad, or from public or private research centers.
L'archive ouverte pluridisciplinaire HAL, est destinée au dépôt et à la diffusion de documents scientifiques de niveau recherche, publiés ou non, émanant des établissements d'enseignement et de recherche français ou étrangers, des laboratoires publics ou privés. 


\title{
Generalized Affine Functions and Generalized Differentials
}

\author{
N. T. H. LINH * $\quad$ J.-P. PENOT ${ }^{\dagger}$
}

\begin{abstract}
We study some classes of generalized affine functions, using a generalized differential. We study some properties and characterizations of these classes and we devise some characterizations of solution sets of optimization problems involving such functions or functions of related classes.

Key words: colinvex, colinfine, generalized differential, optimization problem, protoconvex function, pseudoconvex function, pseudolinear function, quasiconvex function.
\end{abstract}

Mathematics Subject Classification: 26B25, 46G05, 49K27, 90C26, 90C32

\section{Introduction}

A generalized affine function is a function which is both generalized convex and generalized concave. Such functions have been studied in [1], [4], [5], [6], [9], [10], [11], [12], [14], [16], [17], [18], [24]. Among them are quasiaffine functions, i.e. those functions which are both quasiconvex and quasiconcave (see [16], [24]). Also, among them are pseudoaffine functions, i.e. those functions which are differentiable, pseudoconvex and pseudoconcave, also called pseudolinear functions (see [5], [10], [24]). Given a bifunction $h$, the class of $h$-colinfine functions also belongs to this category (see [14]). These references provide interesting, non trivial examples of generalized affine functions; in particular, fractional functions are noticeable pseudo-affine functions and quadratic pseudo-affine functions can be characterized.

It is the purpose of the present paper to introduce and study new concepts of generalized affine functions, as it has been done for generalized convex functions in [15]. Here, to define these classes, we use a generalized differential, i.e. a set-valued map $\partial f$, as a substitute for the derivative of $f$. This concept allows much flexibility as it encompasses several notions of nonsmooth analysis. We give some elementary properties and characterizations for these generalized affine functions. We also present applications to the characterization of the solution set of a constrained minimization problem.

\section{$2 \quad$ Notation and definitions}

Throughout this paper, $X$ is a normed vector space (n.v.s.), $X^{*}$ is the dual space of $X, C$ is a nonempty subset of $X$, and $f: C \rightarrow \mathbb{R}$. We assume that a set-valued map $\partial f: C \rightrightarrows X^{*}$ is given which stands for a substitute to the derivative of $f$; we call it a generalized differential of $f$. As observed in [15], the choice for $\partial f$ is not limited to the subdifferentials of nonsmooth analysis; one can also take the convexificators of [7], the pseudo-differentials of Jeyakumar and Luc ([8]), and much more. We assume that $\partial f(x) \neq \varnothing$ for all $x \in C$ although such an assumption could be relaxed for several results. We denote by $\mathbb{P}$ (resp. $\mathbb{R}_{+}$) the set of positive numbers (resp. non negative numbers) and $\overline{\mathbb{R}}$ (resp. $\mathbb{R}_{\infty}$ ) stands for $\mathbb{R} \cup\{-\infty,+\infty\}$ (resp. $\mathbb{R} \cup\{+\infty\}$ ).

We recall that the visibility cone $V(C, x)$ of $C$ at $x \in C$ is the cone generated by $C-x$ :

$$
V(C, x):=\mathbb{P}(C-x):=\{r(c-x): r \in \mathbb{P}, c \in C\} .
$$

*Laboratoire de Mathématiques, CNRS UMR 5142, Faculté des Sciences, Av. de l'Université 64000 Pau, France (honglinh98t1@yahoo.com)

${ }^{\dagger}$ Laboratoire de Mathématiques, CNRS UMR 5142, Faculté des Sciences, Av. de l'Université 64000 Pau, France (jeanpaul.penot@univ-pau.fr) 
The visibility bundle of $C$ is the set

$$
V C:=\{(x, u) \in C \times X: \exists r \in \mathbb{P}, w \in C, u=r(w-x)\}=\bigcup_{x \in C}\{x\} \times V(C, x) .
$$

It contains the radial tangent bundle of $C$ which is the set

$$
T^{r} C:=\left\{(x, u) \in C \times X: \exists\left(r_{n}\right) \rightarrow 0_{+}, x+r_{n} u \in C \forall n\right\}=\bigcup_{x \in C}\{x\} \times T^{r}(C, x) .
$$

We also use the tangent bundle of $C$ which is the set

$$
T C:=\left\{(x, u) \in C \times X: \exists\left(r_{n}\right) \in \mathbb{P}^{\mathbb{N}},\left(u_{n}\right) \rightarrow u, x+r_{n} u_{n} \in C \forall n\right\}=\bigcup_{x \in C}\{x\} \times T(C, x) .
$$

The upper and the lower radial derivatives (or upper and lower Dini derivatives) of $f$ at $x \in C$ in the direction $u \in T^{r}(C, x)$, are defined by

$$
\begin{aligned}
& D^{+} f(x, u)=\limsup _{t \rightarrow 0+, x+t u \in C} \frac{1}{t}[f(x+t u)-f(x)] ; \\
& D_{+} f(x, u)=\liminf _{t \rightarrow 0+, x+t u \in C} \frac{1}{t}[f(x+t u)-f(x)] .
\end{aligned}
$$

We recall the following definitions (see [15, 20, 24]).

Definition 2.1 Let $f: C \subset X \rightarrow \mathbb{R}$. A function $f$ is said to be

(a) $\partial f$-pseudoconvex at $x$ if for all $w \in C$ :

$$
f(w)<f(x) \Rightarrow \text { for all } x^{*} \in \partial f(x):\left\langle x^{*}, w-x\right\rangle<0 .
$$

(b) $\partial f$-quasiconvex at $x$ if for all $w \in C$ :

$$
f(w)<f(x) \Rightarrow \text { for all } x^{*} \in \partial f(x):\left\langle x^{*}, w-x\right\rangle \leq 0 .
$$

(c) $\partial f$-protoconvex at $x$ if for all $w \in C$ :

$$
f(w) \leq f(x) \Rightarrow \text { for all } x^{*} \in \partial f(x):\left\langle x^{*}, w-x\right\rangle \leq 0 .
$$

We add the word "eventually" when in the preceding implications "for all" is changed into "there exists". Thus, for instance, $f$ is eventually $\partial f$-pseudoconvex at $x$ if for all $w \in C$ satisfying $f(w)<f(x)$ there exists $x^{*} \in \partial f(x)$ such that $\left\langle x^{*}, w-x\right\rangle<0$.

Clearly

$$
f \text { is (eventually) } \partial f \text {-pseudoconvex at } x \Longrightarrow f \text { is (eventually) } \partial f \text {-quasiconvex at } x \text {, }
$$

$$
f \text { is (eventually) } \partial f \text {-protoconvex at } x \Longrightarrow f \text { is (eventually) } \partial f \text {-quasiconvex at } x \text {. }
$$

Now we introduce some definitions related to generalized concavity .

Definition 2.2 Let $f: C \rightarrow \mathbb{R}, g:=-f$ and some $\partial g: C \rightrightarrows X^{*}$ be given. The function $f$ is said to be (eventually) $\partial(-f)$-pseudoconcave (resp. (eventually) $\partial(-f)$-quasiconcave, (eventually) $\partial(-f)$-protoconcave) at $x$ if for $g:=-f$, the function $g$ is (eventually) $\partial g$-pseudoconvex (resp. (eventually) $\partial g$-quasiconvex, (eventually) $\partial g$-protoconvex) at $x$. A function $f: C \rightarrow \mathbb{R}$ is said to be (eventually) $\partial f$-pseudoaffine if it is both (eventually) $\partial f$-pseudoconvex and (eventually) $\partial(-f)$-pseudoconcave. It is said to be $\partial f$-protoaffine if it is $\partial f$-protoconvex and $\partial(-f)$-protoconcave. 
Remark 2.1 (a) If we assume that $\partial(-f)(x)=-\partial f(x)$, then $f$ is $\partial(-f)$-pseudoconcave (resp. $\partial(-f)$ quasiconcave, $\partial(-f)$-protoconcave) at $x$ if and only if $-f$ is $-\partial f$-pseudoconvex (resp. - $\partial f$-quasiconvex, $-\partial f$-protoconvex) at $x$.

(b) When $\partial f(x):=D_{f}(x) \cup-D_{-f}(x)$ where $D_{f}(x)$ and $D_{-f}(x)$ is a subdifferential of $f$ and $-f$ at $x$ respectively, one has $\partial(-f)(x)=-\partial f(x)$.

We recall from [14] that $f: C \rightarrow \mathbb{R}$ is said to be $h$-colinfine at $x \in C$, where $h: V C \rightarrow \overline{\mathbb{R}}$, if there exists $\lambda: C \times C \rightarrow \mathbb{P}$ such that for every $w \in X$,

$$
f(w)-f(x)=\lambda(w, x) h(x, w-x) .
$$

Here we introduce a concept in which the bifunction $h$ is replaced by a generalized differential $\partial f$.

Definition 2.3 (a) A function $f: C \subset X \rightarrow \mathbb{R}$ is $\partial f$-colinfine at $x \in C$ if there exists $\lambda: C \times \operatorname{Graph}(\partial f) \rightarrow \mathbb{P}$ such that for every $w \in C$, for any $x^{*} \in \partial f(x)$

$$
f(w)-f(x)=\lambda\left(w, x, x^{*}\right)\left\langle x^{*}, w-x\right\rangle .
$$

(b) A function $f: C \subset X \rightarrow \mathbb{R}$ is eventually $\partial f$-colinfine at $x \in C$ if there exists $\lambda: C \times \operatorname{Graph}(\partial f) \rightarrow \mathbb{P}$ such that for every $w \in C$, there exists $x^{*} \in \partial f(x)$ satisfying (6).

A function $f$ is said to be $\partial f$-colinfine (resp. $\partial f$-pseudoconvex...) on $C$ if it is $\partial f$-colinfine (resp. $\partial f$ pseudoconvex...) at each point of $C$. For this reason we have chosen to consider $\lambda$ is defined on $C \times \operatorname{Graph}(\partial f)$ in Definition 2.3 rather than on $C \times \partial f(x)$ although only the values of $\lambda$ on $C \times \partial f(x)$ play a role. Similarly, we have chosen to write Definition 2.3(b) in that way in order to stress the analogy with (a). Note that this definition can be rephrased as: $f$ is eventually $\partial f$-colinfine if there exists $\lambda: C \times C \rightarrow \mathbb{P}$ and $g: C \times C \rightarrow X^{*}$ such that for every $w, x \in X, g(w, x) \in \partial f(x)$ and

$$
f(w)-f(x)=\lambda(w, x)\langle g(w, x), w-x\rangle .
$$

When $f$ is differential and $\partial f:=\{D f\}, f$ is $\partial f$-colinfine if, and only if, $f$ is pseudoaffine.

Although the preceding definitions are quite restrictive, they are satisfied in some cases of significant interest.

Note that if $f$ is a $h$-colinfine function, where $h: V C \rightarrow \overline{\mathbb{R}}$ is linear and continuous in its second variable, with $\ell(x):=h(x, \cdot)$ for all $x \in C$, then $f$ is a $\partial f$-colinfine function for $\partial f:=\{\ell\}$ and $f$ is an eventually $\partial f$-colinfine function when $\partial f \supseteq\{\ell\}$. Hence, every pseudoaffine (differentiable) function is $\partial f$-colinfine where $\partial f:=\{D f\}$ or eventually $\partial f$-colinfine where $\partial f \supseteq\{D f\}$. Remark that the converse is not true in general, as shown in Example 2.3, where $f$ is eventually $\partial f$-colinfine but $f$ is not quasiconvex, hence $f$ is not pseudoaffine.

Example 2.1 Let $X=\mathbb{R}^{n}$ and let $a, b \in X^{*}, \alpha, \beta \in \mathbb{R}$. For $C:=\{x \in X: b x+\beta>0\}$, let $f: C \rightarrow \mathbb{R}$ be given by $f(x)=\frac{a x+\alpha}{b x+\beta}$ for all $x \in C$. When $\partial f(x):=\{\nabla f(x)\}, f$ is $\partial f$-colinfine at $x$ since

$$
\nabla f(x)=\frac{(b x+\beta) a+(a x+\alpha) b}{(b x+\beta)^{2}}, \quad f(w)-f(x)=\frac{b x+\beta}{b w+\beta} \nabla f(x)(w-x) .
$$

If $\partial f(x) \supseteq\{\nabla f(x)\}$ then $f$ is eventually $\partial f$-colinfine at $x$.

Example 2.2 Let the function $f: \mathbb{R} \rightarrow \mathbb{R}$ be given by $f(x):=x^{3}$. Then $f$ is not pseudoaffine. But if $\partial f:=\mathbb{P}$ or $\varnothing \neq \partial f \subset \mathbb{P}$, then $f$ is $\partial f$-colinfine. If $\partial f \cap \mathbb{P} \neq \varnothing$ then $f$ is eventually $\partial f$-colinfine.

Example 2.3 Let $f: \mathbb{R} \rightarrow \mathbb{R}$ be given by $f(x)=0$ for $x \neq 0$ and $f(0)=1$. Let $\partial f(x)=\{-1,0,1\}$ for $x \neq 0$ and $\partial f(0)=\{-1,1\}$. Then $f$ is eventually $\partial f$-colinfine but $f$ is not quasiconvex. 
Example 2.4 Let $f: \mathbb{P} \rightarrow \mathbb{R}$ be given by $f(x)=\frac{x^{2}-1}{x}$. Then $\nabla f(x)=\frac{x^{2}+1}{x^{2}}$. If for all $x \in \mathbb{P}, \partial f(x)=$ $\nabla f(x)$ or $\partial f(x) \subseteq \mathbb{P}$ then $f$ is $\partial f$-colinfine. If $\partial f(x) \cap \mathbb{P} \neq \varnothing$ for all $x \in \mathbb{P}$, then $f$ is eventually $\partial f$-colinfine.

Example 2.5 Let $X$ be a n.v.s. and let $g: X \rightarrow \mathbb{R}$ be $\partial g$-colinfine. For $C:=\{x \in X: g(x)>0\}$, let $f(x)=\sqrt{g(x)}$ and $\partial f \subseteq \mathbb{P} \partial g$. $f$ is $\partial f$-colinfine on $C$ since for any $w, x \in C$ and $x_{f}^{*} \in \partial f(x)$, there exist $r \in \mathbb{P}$ and $x_{g}^{*} \in \partial g(x)$ such that $x_{f}^{*}=r x_{g}^{*}$ and

$$
\left\langle x_{f}^{*}, w-x\right\rangle=r\left\langle x_{g}^{*}, w-x\right\rangle=r \frac{g(w)-g(x)}{\lambda\left(w, x, x_{g}^{*}\right)}=r(\sqrt{g(w)}+\sqrt{g(x)}) \frac{f(w)-f(x)}{\lambda\left(w, x, x_{g}^{*}\right)}:=\frac{f(w)-f(x)}{\lambda_{f}\left(w, x, x_{f}^{*}\right)} .
$$

Similarly, if $\partial f \cap \mathbb{P} \partial g \neq \varnothing$ then $f$ is eventually $\partial f$-colinfine on $C$.

Example 2.6 More generally, let $X$ be a n.v.s., $g: X \rightarrow \mathbb{R}$ be $\partial g$-colinfine on $C:=\{x \in X: g(x)>0\}$. Then, for any $p \in \mathbb{N}, f():.=g^{p}():.=(g(.))^{p}$ and $\partial f \subseteq \mathbb{P} \partial g, f$ is $\partial f$-colinfine on $C$ since for any $w, x \in C$ and $x_{f}^{*} \in \partial f(x)$, there exist $r \in \mathbb{P}$ and $x_{g}^{*} \in \partial g(x)$ such that $x_{f}^{*}=r x_{g}^{*}$ and

$$
\begin{aligned}
f(w)-f(x) & =g^{p}(w)-g^{p}(x)=(g(w)-g(x))\left(\sum_{k=0}^{p-1} g^{k}(w) g^{p-k-1}(x)\right) \\
& =\lambda_{g}\left(w, x, x_{g}^{*}\right)\left\langle x_{g}^{*}, w-x\right\rangle\left(\sum_{k=0}^{p-1} g^{k}(w) g^{p-k-1}(x)\right)=\lambda_{f}\left(w, x, x_{f}^{*}\right)\left\langle x_{f}^{*}, w-x\right\rangle
\end{aligned}
$$

where $\lambda_{f}\left(w, x, x_{f}^{*}\right):=r^{-1} \lambda_{g}\left(w, x, x_{g}^{*}\right)\left(\sum_{k=0}^{p-1} g^{k}(w) g^{p-k-1}(x)\right)$. Similarly, if $\partial f \cap \mathbb{P} \partial g \neq \varnothing$ then $f$ is eventually $\partial f$-colinfine on $C$.

The following result is similar to [14, Prop. 4], but instead of using a bifunction as a generalized directional derivative, we use an arbitrary generalized differential. We use a terminology similar to the one in [14, Prop. $4]$ in view of the fact that the vector $v:=\lambda\left(w, x, x^{*}\right)(w-x)$ in the invexity relation (7) is colinear to $w-x$, a feature close to what occurs in the convex case.

Proposition 2.1 For any function $f: C \rightarrow \mathbb{R}, x \in \operatorname{dom} f$ and any multimap $\partial f: C \rightrightarrows X^{*}$, the following assertions are equivalent.

(a) $f$ is $\partial f$-pseudoconvex and $\partial f$-protoconvex at $x \in C$;

(b) $f$ is $\partial f$-colinvex at $x$ in the sense: there exists $\lambda: C \times \operatorname{Graph}(\partial f) \rightarrow \mathbb{P}$ such that for all $w \in C$, $x^{*} \in \partial f(x)$,

$$
f(w) \geq f(x)+\lambda\left(w, x, x^{*}\right)\left\langle x^{*}, w-x\right\rangle .
$$

Proof. (b) $\Rightarrow$ (a) is obvious: given $w \in C$, if $f(w)<f(x)$ then $\left\langle x^{*}, w-x\right\rangle<0$ for all $x^{*} \in \partial f(x)$, while if $f(w) \leq f(x)$ then $\left\langle x^{*}, w-x\right\rangle \leq 0$ for all $x^{*} \in \partial f(x)$. Hence, $f$ is $\partial f$-pseudoconvex and $\partial f$-protoconvex at $x$.

(a) $\Rightarrow$ (b) Let $f$ be $\partial f$-pseudoconvex and $\partial f$-protoconvex at $x$. Let $w \in C$ and $x^{*} \in \partial f(x)$.

If $\left\langle x^{*}, w-x\right\rangle=0$ then one has $f(w) \geq f(x)$ by $\partial f$-pseudoconvexity of $f$ and one can take $\lambda\left(w, x, x^{*}\right)=1$ or any element in $\mathbb{P}$.

If $\left\langle x^{*}, w-x\right\rangle>0$ then $f(w)>f(x)$ by $\partial f$-protoconvexity of $f$. Then one can take $\lambda\left(w, x, x^{*}\right)=$ $\frac{f(w)-f(x)}{\left\langle x^{*}, w-x\right\rangle}>0$.

If $\left\langle x^{*}, w-x\right\rangle<0$ then one can take $\lambda\left(w, x, x^{*}\right)=\max \left\{\frac{f(w)-f(x)}{\left\langle x^{*}, w-x\right\rangle}, 0\right\}+1$, or any $\lambda>0$ such that $\lambda \geq \frac{f(w)-f(x)}{\left\langle x^{*}, w-x\right\rangle}$. For $\left(w, u, u^{*}\right) \in C \times \partial f$ with $u \neq x$ we can take any value for $\lambda\left(w, u, u^{*}\right)$.

A similar result is valid for eventually colinvex functions i.e. functions such that for every $w, x \in C$ there exists $x^{*} \in \partial f(x)$ and $\lambda\left(w, x, x^{*}\right) \in \mathbb{P}$ such that

$$
f(w)-f(x) \geq \lambda\left(w, x, x^{*}\right)\left\langle x^{*}, w-x\right\rangle .
$$


Proposition 2.2 For any function $f: C \rightarrow \mathbb{R}, x \in \operatorname{dom} f$ and any $\partial f: C \rightrightarrows X^{*}$, the following assertions are equivalent.

(a) $f$ is eventually $\partial f$-pseudoconvex and eventually $\partial f$-protoconvex at $x$;

(b) $f$ is eventually $\partial f$-colinvex at $x$.

\section{Characterizations of generalized affine functions}

In the present section we study classes of generalized affine functions which are still more restrictive. Their interests lie in their striking properties. In particular, they enjoy nice composition properties with the classes introduced in the preceding section. Their behaviors will be studied below.

First, we shall consider some composition properties.

Proposition 3.1 Let $g: C \rightarrow \mathbb{R}$ be (eventually) $\partial g$-colinfine. Let $\varphi: \mathbb{R} \rightarrow \mathbb{R}$ and $f:=\varphi \circ g$ and let $\partial f(x):=$ $\partial \varphi(g(x)) \partial g(x)$.

(a) If $\varphi$ is (eventually) $\partial \varphi$-pseudoconvex, then $f$ is (eventually) $\partial f$-pseudoconvex;

(b) If $\varphi$ is (eventually) $\partial \varphi$-quasiconvex, then $f$ is (eventually) $\partial f$-quasiconvex;

(c) If $\varphi$ is (eventually) $\partial \varphi$-protoconvex, then $f$ is (eventually) $\partial f$-protoconvex;

(d) If $\varphi$ is (eventually) $\partial \varphi$-colinfine, then $f$ is (eventually) $\partial f$-colinfine.

Proof. Since $g$ is $\partial g$-colinfine, for all $w, x \in C$ and all $x^{*} \in \partial g(x)$ there exists $\lambda_{g}\left(w, x, x^{*}\right) \in \mathbb{P}$ such that $g(w)-g(x)=\lambda_{g}\left(w, x, x^{*}\right)\left\langle x^{*}, w-x\right.$. Let $u:=\varphi(x)$ and $v:=\varphi(w)$.

(a) Since $\varphi$ is $\partial \varphi$-pseudoconvex, if there exists $x_{f}^{*} \in \partial f(x)$ such that $\left\langle x_{f}^{*}, w-x\right\rangle \geq 0$ then there exist $x_{g}^{*} \in \partial g(x)$ and $u^{*} \in \partial \varphi(u)$ such that $x_{f}^{*}=u^{*} x_{g}^{*} \geq 0$. Moreover,

$$
x_{f}^{*}=u^{*} x_{g}^{*} \geq 0 \Rightarrow u^{*}(g(w)-g(x)) \geq 0 \Rightarrow \varphi(g(w)) \geq \varphi(g(x)) .
$$

(b) Since $\varphi$ is $\partial \varphi$-quasiconvex, if there exists $x_{f}^{*} \in \partial f(x)$ such that $\left\langle x_{f}^{*}, w-x\right\rangle>0$ then there exist $x_{g}^{*} \in \partial g(x)$ and $u^{*} \in \partial \varphi(u)$ such that $x_{f}^{*}=u^{*} x_{g}^{*}>0$. Moreover,

$$
x_{f}^{*}=u^{*} x_{g}^{*}>0 \Rightarrow u^{*}(g(w)-g(x))>0 \Rightarrow \varphi(g(w)) \geq \varphi(g(x)) .
$$

(c) A similar proof can be given when $\varphi$ is $\partial \varphi$-protoconvex.

(d) Now, if $\varphi$ is $\partial \varphi$-colinfine, then for all $x_{f}^{*} \in \partial f(x)$, there exist some $x_{g}^{*} \in \partial g(x)$ and some $u^{*} \in \partial \varphi(u)$ such that $x_{f}^{*}=u^{*} x_{g}^{*}$. Moreover, one has

$$
\varphi(g(w))-\varphi(g(x))=\lambda_{\varphi}\left(v, u, u^{*}\right)\left\langle u^{*}, g(w)-g(x)\right\rangle=\lambda_{\varphi}\left(v, u, u^{*}\right) \lambda_{g}\left(w, x, x_{g}^{*}\right)\left\langle u^{*} x_{g}^{*}, w-x\right\rangle
$$

hence $f$ is $\partial f$-colinfine.

When $g$ is eventually $\partial g$-colinfine, for $w, x \in C$ there exist $x_{g}^{*} \in \partial g(x)$ and $\lambda_{g}\left(w, x, x^{*}\right) \in \mathbb{P}$ such that $g(w)-g(x)=\lambda_{g}\left(w, x, x^{*}\right)\left\langle x_{g}^{*}, w-x\right\rangle$. Let $u:=\varphi(x)$ and $v:=\varphi(w)$.

Let $\varphi$ be eventually $\partial \varphi$-pseudoconvex. If $\varphi(g(w)) \leq \varphi(g(x))$, then there exists $u^{*} \in \partial \varphi(u)$ such that $\left\langle u^{*}, v-u\right\rangle \leq 0$. Hence

$$
\varphi(g(w)) \leq \varphi(g(x)) \Rightarrow\left\langle u^{*}, g(w)-g(x)\right\rangle \leq 0 \Rightarrow\left\langle u^{*} x_{g}^{*}, w-x\right\rangle \leq 0 .
$$

Clearly, $u^{*} x_{g}^{*} \in \partial f(x)$. Thus, $f$ is eventually $\partial f$-pseudoconvex.

Similar proofs can be given for (b), (c) and (d).

From the preceding proposition and Definition 2.3, we have the following example show simple contructions of colinfine functions.

Example 3.1 Let $g, h: C \rightarrow \mathbb{R}, g$ be $\partial g$-colinfine and $h$ be $\partial h$-colinfine and let $x \in C$.

(a) Let $f:=g+h$ or $f:=g h$. If $\mathbb{P} \partial g=\mathbb{P} \partial h$ and if $\partial f \subseteq \mathbb{P} \partial g$, then $f$ is $\partial f$-colinfine.

(b) Let $f(\cdot):=\frac{1}{g(\cdot)}$ and $D:=\{x \in C: g(x)>0\}$. If $\partial f \subseteq-\mathbb{P} \partial g$, then $f$ is $\partial f$-colinfine on $D$. 
Let us turn to properties of colinfine functions. The following result is an easy consequence of Lemma 2.1 and Lemma 2.2 .

Proposition 3.2 (a) A function $f$ is $\partial f$-colinfine if, and only if, $f$ is $\partial f$-colinvex and $-f$ is $-\partial f$-colinvex.

(b) Suppose that for all $x \in C$ the set $\partial f(x)$ is convex. If $f$ is eventually $\partial f$-colinvex and $-f$ is eventually $-\partial f$-colinvex, then $f$ is eventually $\partial f$-colinfine.

(c) Let $C$ be open and let $f$ be radially continuous. If $f$ is (eventually) $\partial f$-protoconvex and $-f$ is (eventually) $-\partial f$-protoconvex and $0 \notin \partial f(x)$ for all $x \in C$ then $f$ is (eventually) $\partial f$-colinfine.

Proof. (a) Let $f$ be $\partial f$-colinfine. Then, there exists $\lambda: C \times \operatorname{Graph}(\partial f) \rightarrow \mathbb{P}$ such that for all $w, x \in C$, $x^{*} \in \partial f(x)$,

$$
f(w)-f(x)=\lambda\left(w, x, x^{*}\right)\left\langle x^{*}, w-x\right\rangle .
$$

Thus, $f$ is $\partial f$-colinvex and $-f$ is $-\partial f$-colinvex since this function $\lambda$ will satisfy relation (7) both for $f$ and $-f$.

Conversely, let $w, x \in C$. Since $f$ is $\partial f$-colinvex and $-f$ is $-\partial f$-colinvex, there exist $\lambda_{1}: C \times \operatorname{Graph}(\partial f) \rightarrow \mathbb{P}$ and $\lambda_{2}: C \times \operatorname{Graph}(\partial f) \rightarrow \mathbb{P}$ such that for all $w, x \in C, x^{*} \in \partial f(x)$,

$$
\begin{gathered}
f(w)-f(x) \geq \lambda_{1}\left(w, x, x^{*}\right)\left\langle x^{*}, w-x\right\rangle, \\
-f(w)+f(x) \geq \lambda_{2}\left(w, x, x^{*}\right)\left\langle-x^{*}, w-x\right\rangle .
\end{gathered}
$$

Thus, if $f(w)-f(x)<0$ then $\left\langle x^{*}, w-x\right\rangle<0$, while if $f(w)-f(x)>0$ then $\left\langle x^{*}, w-x\right\rangle>0$, hence in both cases we can take $\lambda\left(w, x, x^{*}\right)=\frac{f(w)-f(x)}{\left\langle x^{*}, w-x\right\rangle}>0$. Now when $f(w)=f(x)$ then $\left\langle x^{*}, w-x\right\rangle=0$ and one can take any $\lambda\left(w, x, x^{*}\right) \in \mathbb{P}$.

(b) If $f$ is eventually $\partial f$-colinfine, then as in the proof of (a), one gets that $f$ is eventually $\partial f$-colinvex and $-f$ is eventually $-\partial f$-colinvex.

Conversely, let $w, x \in C$. Since $f$ is eventually $\partial f$-colinvex and $-f$ is eventually $-\partial f$-colinvex, there exist $\lambda_{1}: C \times C \rightarrow \mathbb{P}, \lambda_{2}: C \times C \rightarrow \mathbb{P}$ and $g_{1}: C \times C \rightarrow X^{*}, g_{2}: C \times C \rightarrow X^{*}$ such that for all $w, x \in C$,

$$
\lambda_{2}(w, x)\left\langle g_{2}(w, x), w-x\right\rangle \geq f(w)-f(x) \geq \lambda_{1}(w, x)\left\langle g_{1}(w, x), w-x\right\rangle .
$$

If $f(w)-f(x)<0$ then $\left\langle g_{1}(w, x), w-x\right\rangle<0$, hence we can take $g(w, x):=g_{1}(w, x), \lambda(w, x)=\frac{f(w)-f(x)}{\left\langle g_{1}(w, x), w-x\right\rangle}>$ 0 ; if $f(w)-f(x)>0$ then $\left\langle g_{2}(w, x), w-x\right\rangle>0$ and we can take $g(w, x):=g_{2}(w, x), \lambda(w, x)=\frac{f(w)-f(x)}{\left\langle g_{2}(w, x), w-x\right\rangle}>$ 0 , while if $f(w)=f(x)$ then $\left\langle g_{1}(w, x), w-x\right\rangle \leq 0$ and $\left\langle g_{2}(w, x), w-x\right\rangle \geq 0$ and, since $\partial f(x)$ is convex, there exists $g_{3}(w, x) \in X^{*}$ such that $\left\langle g_{3}(w, x), w-x\right\rangle=0$ and we can take $g(w, x):=g_{3}(w, x)$ and any $\lambda\left(w, x, x^{*}\right) \in \mathbb{P}$.

(c) Under the assumptions of (c), $f$ is $\partial f$-pseudoconvex and $-f$ is $-\partial f$-pseudoconvex (see [15, Proposition 2.5]). By Proposition 2.1, $f$ is $\partial f$-colinvex and $-f$ is $-\partial f$-colinvex. Hence from (a) $f$ is $\partial f$-colinfine.

Similarly, if $f$ is eventually $\partial f$-protoconvex and $-f$ is eventually $-\partial f$-protoconvex and $0 \notin \partial f(x)$ for all $x \in C$ then $f$ is (eventually) $\partial f$-colinfine.

It may happen that $f$ is eventually $\partial f$-colinvex and $-f$ is eventually $-\partial f$-colinvex but $f$ is not eventually $\partial f$-colinfine when $\partial f$ is not convex.

Example 3.2 Let $f: \mathbb{R} \rightarrow \mathbb{R}$ be given by $f(x)=0$ for $x \leq 0$ and $f(x)=x$ for $x \geq 0$ and let $\partial f$ be given by $\partial f(x)=\{-1,1\}$ for all $x \in C:=X$. Then $f$ is eventually $\partial f$-colinvex and $-f$ is eventually $-\partial f$-colinvex but $f$ is not eventually $\partial f$-colinfine since $f(-1)=f(0)=0$ and $\langle 1,-1-0\rangle \neq 0,\langle-1,-1-0\rangle \neq 0$.

Proposition 3.3 (a) If $f$ is $\partial f$-colinfine on $C$, then $f$ is quasiaffine (i.e. quasiconvex and quasiconcave) on $C$.

(b) If $f$ is $\partial f$-colinfine at $x \in C$, then, setting $\partial(-f)(x):=-\partial f(x), f$ is $\partial f$-protoaffine and $\partial f$-pseudoaffine at $x$. 
Proof. (a) Suppose that $f$ is not quasiaffine: there exist $x, y, z \in C$ with $y \in] x, z[$ such that either $f(y)>\max \{f(x), f(z)\}$ or $f(y)<\min \{f(x), f(z)\}$. If $f(y)>\max \{f(x), f(z)\}$ then for $y^{*} \in \partial f(y)$, one has $\left\langle y^{*}, x-y\right\rangle<0$ and $\left\langle y^{*}, z-y\right\rangle<0$, a contradiction with the linearity of $y^{*}$. If $f(y)<\min \{f(x), f(z)\}$ a similar proof yields a contradiction. 2.1 .

(b) Since $f$ is $\partial f$-colinvex and $-f$ is $-\partial f$-colinvex, the assertion follows from Remark 1 and Proposition

Now, let us give a partial converse of the preceding result. It uses the following assumptions in which $x \in C$ :

$$
\begin{array}{rlc}
\forall u \in T^{r}(C, x) & D^{+} f(x, u)>0, & D_{+} f(x, u) \geq 0 \Rightarrow D_{+} f(x, u)>0, \\
\forall x^{*} \in \partial f(x) \quad \forall u \in T^{r}(C, x) & \left\langle x^{*}, u\right\rangle \leq D^{+} f(x, u) \\
\forall x^{*} \in \partial(-f)(x) \quad \forall u \in T^{r}(C, x) & \left\langle x^{*}, u\right\rangle \leq D^{+}(-f)(x, u) \\
\forall x^{*} \in \partial f(x) \quad \forall u \in T^{r}(C, x) & D_{+} f(x, u) \leq\left\langle x^{*}, u\right\rangle
\end{array}
$$

Obviously, condition (8) is satisfied when $f$ has a radial derivative at $x$ in all directions $u \in T^{r}(C, x)$. Moreover, if $C$ is open, $f$ is differentiable on $C$ and $\partial f(x)=\left\{f^{\prime}(x)\right\}, \partial(-f)(x)=\left\{-f^{\prime}(x)\right\}$, the other conditions are satisfied. However, these conditions allow $x$ to be a boundary point of $C$ (which is not assumed to be open in the next results).

Let us recall that a function $f$ is radially nonconstant if one cannot find any proper line segment on which $f$ is constant.

Proposition 3.4 Let $f$ be $\partial f$-pseudoaffine on a convex set $C$. Then $f$ is quasiaffine on $C$. Let $x \in C$.

(a) If $f$ is radially nonconstant and if $\partial(-f)=-\partial f$, then $f$ is $\partial f$-colinfine.

(b) If relation (9) is satisfied, then $f$ is $\partial f$-colinvex at $x$.

In addition to (9), if one of the following two conditions holds, then $f$ is $\partial f$-colinfine at $x$.

(i) $\partial(-f)(x)=-\partial f(x)$ and relation (10) is satisfied,

(ii) conditions (8), (11) are satisfied at $x$ and for all $u \in V(C, x)$, there exists $y^{*} \in \partial(-f)(x)$ such that $D_{+}(-f)(x, u) \leq\left\langle y^{*}, u\right\rangle$.

Proof. Since $f$ is $\partial f$-pseudoconvex and $-f$ is $\partial(-f)$-pseudoconvex, $f$ and $-f$ are quasiconvex by [15, Proposition 6]. Thus, $f$ is quasiaffine on $C$.

(a) Let us first prove that $f$ is $\partial f$-protoconvex. Suppose that $f$ is not $\partial f$-protoconvex. Then there exist some $w, x \in C$ such that $f(w) \leq f(x)$ and some $x^{*} \in \partial f(x)$ with $\left\langle x^{*}, w-x\right\rangle>0$. Then $f(w)=f(x)$. On the other hand, since $f$ is quasiconvex and radially nonconstant, there exists $z \in(w, x)$ such that $f(z)<f(x)$ and then $\left\langle x^{*}, z-x\right\rangle<0$, a contradition.

Similarly, if $-f$ is not $\partial(-f)$-protoconvex, then there exist some $w, x \in C$ such that $-f(w) \leq-f(x)$ and some $x^{*} \in \partial(-f)(x)$ with $\left\langle x^{*}, w-x\right\rangle>0$. Since $-f$ is $\partial(-f)$-pseudoconvex, one has $f(w)=f(x)$. Since $f$ is quasiconcave and radially nonconstant, there exists $z \in(w, x)$ such that $f(z)>f(x)$, i.e. $-f(z)<-f(x)$ and then $\left\langle x^{*}, z-x\right\rangle<0$, a contradition. Thus, $-f$ is $\partial(-f)$-protoconvex.

On the other hand, since $\partial(-f)=-\partial f,-f$ is $-\partial f$-protoconvex and $-f$ is $-\partial f$-pseudoconvex. Hence, $f$ is $\partial f$-colinvex and $-f$ is $-\partial f$-colinvex. By Proposition $3.2(\mathrm{a}), f$ is $\partial f$-colinfine at $x$.

(b) When relation (9) holds, we see that $f$ is $\partial f$-protoconvex at $x$, since if $f(w) \leq f(x)$ for some $w \in C$, then $D^{+} f(x, w-x) \leq 0$ by quasiconvexity and for all $x^{*} \in \partial f(x)$ we have $\left\langle x^{*}, w-x\right\rangle \leq D^{+} f(x, w-x) \leq 0$. Thus, since $f$ is $\partial f$-pseudoconvex, it is $\partial f$-colinvex at $x$ by Proposition 2.1.

Let us first consider the case (i): $\partial(-f)(x)=-\partial f(x)$ and relation (10) is satisfied. Since $f$ is $\partial f$ pseudoaffine, $f$ is $\partial(-f)$-pseudoconcave, hence by Definition $2.2,-f$ is $-\partial f$-pseudoconvex.

Now, let us prove that $f$ is $\partial(-f)$-protoconcave at $x$. If $-f(w) \leq-f(x)$ for some $w \in C$, then for all $x^{*} \in \partial(-f)(x)$ one has $\left\langle x^{*}, w-x\right\rangle \leq D^{+}(-f)(x, w-x) \leq 0$ by relation $(10)$ and the fact that $-f$ is quasiconvex. Thus, $-f$ is $-\partial f$-protoconvex at $x$ and $-f$ is $-\partial f$-colinvex at $x$. By Proposition 3.2 (a), $f$ is $\partial f$-colinfine at $x$.

Let us suppose assumption (ii) is satisfied. 
Let us prove that $-f$ is $-\partial f$-protoconvex if relation (11) is satisfied. If $-f(w) \leq-f(x)$ for some $w \in C$, then $f(w) \geq f(x)$ and $D_{+} f(x, w-x) \geq 0$ since $f$ is quasiaffine. For any $x^{*} \in \partial f(x)$ relation (11) implies $0 \leq D_{+} f(x, w-x) \leq\left\langle x^{*}, w-x\right\rangle$. Thus one has $\left\langle-x^{*}, w-x\right\rangle \leq 0$ and $-f$ is $-\partial f$-protoconvex at $x$.

Now, let us prove that $-f$ is $-\partial f$-pseudoconvex. Since $f$ is $\partial f$-pseudoaffine, $-f$ is $\partial(-f)$-pseudoconvex. Let $w \in C$ be such that $-f(w)<-f(x)$ and let $u:=w-x$. Then we have $\left\langle y^{*}, w-x\right\rangle<0$ for all $y^{*} \in \partial(-f)(x)$. Let us pick $y^{*} \in \partial(-f)(x)$ such that $D_{+}(-f)(x, u) \leq\left\langle y^{*}, u\right\rangle$. Thus, we have $D^{+} f(x, w-x)>0$. Since $f$ is quasiaffine and $f(w)>f(x)$, we also have $D_{+} f(x, w-x) \geq 0$. Then condition (8) entails $0<D_{+} f(x, w-x)$. Since relation (11) is satisfied, for all $x^{*} \in \partial f(x)$ we have $0<D_{+} f(x, w-x) \leq\left\langle x^{*}, w-x\right\rangle$. Thus, we get $\left\langle-x^{*}, w-x\right\rangle<0$ for all $-x^{*} \in-\partial f(x)$ and then $-f$ is $-\partial f$-pseudoconvex.

Thus, $-f$ is $-\partial f$-colinvex at $x$ and then $f$ is colinfine at $x$.

Let us give a similar result for an eventually colinfine function at some $x \in C$. It uses the following assumptions which are weaker than the preceding assumptions:

$$
\begin{aligned}
& \forall u \in T^{r}(C, x) \quad \exists x^{*} \in \partial f(x) \quad\left\langle x^{*}, u\right\rangle \leq D^{+} f(x, u) \\
& \forall u \in T^{r}(C, x) \quad \exists x^{*} \in \partial(-f)(x) \quad\left\langle x^{*}, u\right\rangle \leq D^{+}(-f)(x, u) \\
& \forall u \in T^{r}(C, x) \quad \exists x^{*} \in \partial f(x) \quad D_{+} f(x, u) \leq\left\langle x^{*}, u\right\rangle
\end{aligned}
$$

Proposition 3.5 Let $f$ be $\partial f$-pseudoaffine on a convex set $C$ and let $x \in C$.

(a) If $f$ is radially nonconstant and if $\partial(-f) \cap-\partial f \neq \varnothing$, then $f$ is eventually $\partial f$-colinfine.

(b) If relation to (12) is satisfied, then $f$ is eventually $\partial f$-colinvex.

In addition, if $\partial f(x)$ is convex and one of the following conditions holds, then $f$ is eventually $\partial f$-colinfine at $x$.

(i) $\partial(-f)(x) \cap-\partial f(x) \neq \varnothing$ and relation (10) is satisfied,

(ii) $\partial(-f)(x)=-\partial f(x)$ and relation (13) is satisfied,

(iii) conditions (8), (14) are satisfied at $x$ and for all $u \in T^{r}(C, x)$ there exists $y^{*} \in \partial(-f)(x)$ such that $D_{+}(-f)(x, u) \leq\left\langle y^{*}, u\right\rangle$.

Proof. By the first assertion of the preceding proposition, $f$ is quasiaffine on $C$.

(a) As in the proof of the preceding proposition (a), $f$ is $\partial f$-protoconvex and $-f$ is $\partial(-f)$-protoconvex. Moreover, since $\partial(-f) \cap-\partial f \neq \varnothing,-f$ is eventually $-\partial f$-protoconvex and $-f$ is eventually $-\partial f$-pseudoconvex. Hence, $f$ is $\partial f$-colinvex and $-f$ is eventually $-\partial f$-colinvex. By Proposition $3.2(\mathrm{~b}), f$ is eventually $\partial f$-colinfine at $x$.

(b) Let us first observe that $f$ is eventually $\partial f$-protoconvex at $x$, since if $f(w) \leq f(x)$ for some $w \in C$, then $D^{+} f(x, w-x) \leq 0$, and, by relation (12), there exists $x^{*} \in \partial f(x)$ such that $\left\langle x^{*}, w-x\right\rangle \leq D^{+} f(x, w-x) \leq 0$. Since $f$ is also $\partial f$-pseudoconvex, it is eventualy $\partial f$-colinvex at $x$ by Proposition 2.2 .

In order to show that $f$ is eventually $\partial f$-colinfine at $x$, let us first consider the case (i). Since $f$ is $\partial f$ pseudoaffine, $f$ is $\partial(-f)$-pseudoconcave, hence by Definition $2.2,-f$ is $\partial(-f)$-pseudoconvex. Since relation (10) is satisfied, as in the proof of the preceding proposition, we get that $-f$ is $\partial(-f)$-protoconvex at $x$. Thus, $-f$ is $\partial(-f)$-colinvex. On the other hand, since $\partial(-f)(x) \cap-\partial f(x) \neq \varnothing,-f$ is eventually $-\partial f$-pseudoconvex and eventually $-\partial f$-protoconvex at $x$. Thus, $f$ is eventually $\partial f$-colinfine at $x$ by Proposition 3.2 (b).

Now, let us suppose assumption (ii) is satisfied. First, we observe that $f$ is eventually $\partial(-f)$-protoconcave at $x$ : since if $-f(w) \leq-f(x)$ for some $w \in C$, then for some $x^{*} \in \partial(-f)(x)$ one has $\left\langle x^{*}, w-x\right\rangle \leq D^{+}(-f)(x, w-$ $x) \leq 0$ by relation (13). On the other hand, since $\partial(-f)(x)=-\partial f(x),-f$ is eventually $-\partial f$-protoconvex at $x$. So, $f$ is eventually $\partial f$-colinfine at $x$.

Finally, let us suppose assumption (iii) is satisfied. Let us first prove that $-f$ is eventually $-\partial f$-protoconvex at $x$. If $-f(w) \leq-f(x)$ for some $w \in C$, then $f(w) \geq f(x)$ and $D_{+} f(x, w-x) \geq 0$ since $f$ is quasiaffine. By relation (14), there exists $x^{*} \in \partial f(x)$ such that $0 \leq D_{+} f(x, w-x) \leq\left\langle x^{*}, w-x\right\rangle$. Thus one has $-x^{*} \in-\partial f(x)$ with $\left\langle-x^{*}, w-x\right\rangle \leq 0$ : $-f$ is eventually $-\partial f$-protoconvex at $x$.

Now, let us prove that $-f$ is eventually $-\partial f$-pseudoconvex at $x$. Since $f$ is $\partial f$-pseudoaffine, $-f$ is $\partial(-f)$ pseudoconvex. If $-f(w)<-f(x)$ for some $w \in C$, using our assumption and condition (8) as in the preceding proof, we get $0<D_{+} f(x, w-x)$. Since relation (14) is satisfied, there exists $x^{*} \in \partial f(x)$ such that $D_{+} f(x, w-$ 
$x) \leq\left\langle x^{*}, w-x\right\rangle$. Thus, we get $-x^{*} \in-\partial f(x)$ with $\left\langle-x^{*}, w-x\right\rangle<0$ : $-f$ is eventually $-\partial f$-pseudoconvex at $x$.

Thus, $-f$ is eventually $-\partial f$-colinvex at $x$ and then $f$ is eventually $\partial f$-colinfine at $x$.

Remark 3.1 (a) Note that if we change " $\leq$ " into " $<$ " in relations (11), (9), then assumption (8) in the preceding results could be deleted.

(b) Clearly, condition (8) is satisfied if $f$ has a radial derivative in the direction $u$ for all $u \in T^{r}(C, x)$.

(c) The preceding proposition implies [14, Proposition 13].

Proposition 3.6 Let $f$ be $\partial f$-colinfine on $C$ and let $w, x \in C$. For $r \in \mathbb{R}$, let $x_{r}:=x+r(w-x)$. Then for any $s, t \in \mathbb{R}$, with $s \neq t$ such that $x_{s}, x_{t} \in C$, the following statements are equivalent:

(a) $f(x)=f(w)$;

(b) $f\left(x_{t}\right)=f\left(x_{s}\right)$;

(c) $\left\langle x_{t}^{*}, x_{s}-x_{t}\right\rangle=0$ for all $x_{t}^{*} \in \partial f\left(x_{t}\right)$;

(d) $\left\langle x_{s}^{*}, x_{t}-x_{s}\right\rangle=0$ for all $x_{s}^{*} \in \partial f\left(x_{s}\right)$.

Proof. (a) $\Rightarrow$ (b) If $f(x)=f(w)$ then for any $x^{*} \in \partial f(x),\left\langle x^{*}, w-x\right\rangle=0$ and then, $0=\left\langle x^{*}, x_{t}-x\right\rangle=$ $\left\langle x^{*}, x_{s}-x\right\rangle$. By Definition 2.3, $f\left(x_{t}\right)=f(x)=f\left(x_{s}\right)$.

$(\mathrm{b}) \Leftrightarrow(\mathrm{c}) \Leftrightarrow(\mathrm{d})$ is obvious by Definition 2.3 .

(c) $\Rightarrow$ (a) If $\left\langle x_{t}^{*}, x_{s}-x_{t}\right\rangle=0$ for all $x_{t}^{*} \in \partial f\left(x_{t}\right)$ then, since $s \neq t$, for all $x_{t}^{*} \in \partial f\left(x_{t}\right)$ one has $\left\langle x_{t}^{*}, x-w\right\rangle=0$, hence $\left\langle x_{t}^{*}, x-x_{t}\right\rangle=\left\langle x_{t}^{*}, w-x_{t}\right\rangle=0$ and then $f(x)=f\left(x_{t}\right)=f(w)$.

For an eventually colinfine function, we have the following property in the spirit of Minty variational inequalities and Stampachia variational inequalities. It shows that, in our case, a solution in the sense of Minty is a solution in the sense of Stampachia.

Recall ([2]) that a multimap $T: C \rightrightarrows X^{*}$ is said to be upper sign-continuous on $C$ if, for every $w, x \in C$, the following implication (in which $x_{t}:=t x+(1-t) w$ ) holds:

$$
\forall t \in(0,1), \quad \inf \left\{\left\langle x_{t}^{*}, w-x\right\rangle: x_{t}^{*} \in T\left(x_{t}\right)\right\} \geq 0 \Rightarrow \sup \left\{\left\langle x^{*}, w-x\right\rangle: x^{*} \in T(x)\right\} \geq 0 .
$$

Proposition 3.7 Let $f$ be eventually $\partial f$-colinfine on $C$ and let $w, x \in C$.

(a) If for all $w^{*} \in \partial f(w)$ one has $\left\langle w^{*}, w-x\right\rangle \geq 0$ (resp. $\left\langle w^{*}, w-x\right\rangle>0,\left\langle w^{*}, w-x\right\rangle=0,\left\langle w^{*}, w-x\right\rangle<0$, $\left.\left\langle w^{*}, w-x\right\rangle \leq 0\right)$ then there exists $x^{*} \in \partial f(x)$ such that $\left\langle x^{*}, w-x\right\rangle \geq 0$ (resp. $\left\langle x^{*}, w-x\right\rangle>0,\left\langle x^{*}, w-x\right\rangle=0$, $\left.\left\langle x^{*}, w-x\right\rangle<0,\left\langle x^{*}, w-x\right\rangle \leq 0\right)$.

(b) $\partial f$ is upper sign-continuous on $C$.

Proof. (a) Since $f$ is eventually $\partial f$-colinfine on $C$, there exist $w^{*} \in \partial f(w), x^{*} \in \partial f(x), \lambda\left(w, x, x^{*}\right) \in \mathbb{P}$ and $\lambda\left(x, w, w^{*}\right) \in \mathbb{P}$ such that

$$
f(w)-f(x)=\lambda\left(w, x, x^{*}\right)\left\langle x^{*}, w-x\right\rangle=-\lambda\left(x, w, w^{*}\right)\left\langle w^{*}, x-w\right\rangle .
$$

If for all $w^{*} \in \partial f(w)$ one has $\left\langle w^{*}, w-x\right\rangle \geq 0$, then the preceding relation entails $f(w) \geq f(x)$. Thus, $\left\langle x^{*}, w-x\right\rangle \geq 0$. The proofs of the other assertions are similar.

(b) Since $x_{t}-x=t(w-x)$ for $t \in(0,1)$, (b) follows from (a) by substituting $x_{t}, x_{t}^{*}$ to $w, w^{*}$.

The preceding proposition can easily adapted to the case $f$ is $\partial f$-colinfine by interchanging "for all" and "there exists". Moreover, in such a case, one sees $\partial f$ and $-\partial f$ are pseudomonotone (in the sense of [26]; see also [20], [25]). Thus we get a multivalued generalization of the concept of PPM map which has been used in [3] to study variational inequalities.

The following result is similar to [14, Proposition 10].

Proposition 3.8 If $f$ is $\partial f$-colinfine then the function $t \mapsto f(x+t(w-x))$ is either increasing or decreasing or constant on the interval on which it is defined. 
Proof. Since $f$ is $\partial f$-colinfine, there exists $\lambda: C \times \operatorname{Graph}(\partial f) \rightarrow \mathbb{P}$ such that for every $w, x \in C$, for any $x^{*} \in \partial f(x)$,

$$
f(w)-f(x)=\lambda\left(w, x, x^{*}\right)\left\langle x^{*}, w-x\right\rangle .
$$

If $f(w)=f(x)$ then by Proposition 3.6, one has $f(x+t(w-x))=f(x)$ for all $t \in \mathbb{R}$ such that $x_{t}:=$ $x+t(w-x) \in C$.

If $f(w)>f(x)$ then for any $x^{*} \in \partial f(x)$ one has $\left\langle x^{*}, w-x\right\rangle>0$. For all $t>0$ such that $x_{t}:=x+t(w-x) \in C$, by homogeneity, one gets $\left\langle x^{*}, x_{t}-x\right\rangle>0$. Thus $f\left(x_{t}\right)>f(x)$. Also, given $u:=x+r(w-x) \in C$ and $v:=x+s(w-x) \in C$ with $s>r$, one has $f(v)>f(u)$. Otherwise, one would have either $f(v)=f(u)$ and then $f(w)=f(x)$ by what precedes, or $f(v)<f(u)$ and then, for all $v^{*} \in \partial f(v),\left\langle v^{*}, u-v\right\rangle>0$, hence $\left\langle v^{*}, x-v\right\rangle>0$ since $x-v=q(u-v)$ for some $q>0$ and then $f(x)>f(v)$, a contradiction with $f(v)=f\left(x_{s}\right)>f(x)$. A similar proof shows that for every $t>0$ we have $f(w+t(x-w))<f(w)$ and for $s>r, f(w+s(x-w))<f(w+r(x-w))$ when the involved points are in $C$.

If $f(x)>f(w)$, then we also have the conclusion by interchanging the roles of $w$ and $x$ in what precedes. $\square$

In the sequel, we will consider the following hypothesis:

$\left(\mathrm{H}^{+}\right)$If $\left\langle x^{*}, w-x\right\rangle>0$ for some $w, x \in C$ and some $x^{*} \in \partial f(x)$, then there exists $\left.z \in\right] w, x[$ such that $f(z)>f(x)$.

$\left(\mathrm{H}^{-}\right)$If $\left\langle x^{*}, w-x\right\rangle<0$ for some $w, x \in C$ and some $x^{*} \in \partial f(x)$, then there exists $\left.z \in\right] w, x[$ such that $f(z)<f(x)$.

$\left(\mathrm{H}_{0}\right)$ If $\left\langle x^{*}, w-x\right\rangle=0$ for some $w, x \in C$ and some $x^{*} \in \partial f(x)$, then one has $f(w)=f(x)$.

Hypothesis $\left(\mathrm{H}^{+}\right)$(resp. $\left.\left(\mathrm{H}^{-}\right)\right)$is weaker than $\partial f$-protoconvexity of $f$ (resp. $-\partial f$-protoconvexity of $-f$ ). Hypothesis $\left(\mathrm{H}^{+}\right)$(resp. $\left(\mathrm{H}^{-}\right)$) is clearly satisfied when $\partial f(x)$ (resp. $\left.-\partial f(x)\right)$ is contained in the Dini-Hadamard (or contingent) subdifferential (resp. $\partial^{D}(-f)(x)$ of $-f$ at $x$ ).

The following theorem has been given in [5, Theorems 4.13, 4.14], [6], [10], [11], in the case the function $f$ is differentiable and $\partial f:=\left\{f^{\prime}\right\}$. Here, the function $f$ is nonsmooth. In [14], a generalized directional derivative is used instead of a generalized differential. We start with a preparatory lemma dealing with the one-dimensional case.

Lemma 3.1 Let $C$ be an open interval of $\mathbb{R}$ and let $\varphi: C \rightarrow \mathbb{R}$ be a continuous function satisfying $\left(H^{+}\right)$ and $\left(H^{-}\right)$with respect to $\partial \varphi$. Then $\varphi$ is $\partial \varphi$-colinfine if, and only if, either $\left(H_{0}\right)$ is satisfied and there exists some $t_{0} \in C$ such that $\partial \varphi\left(t_{0}\right) \ni\{0\}$, or for all $t \in C$ the sign of $t^{*} \in \partial \varphi(t)$ is constant on the level set $L_{\varphi}(t):=\varphi^{-1}(\varphi(t))$.

Proof. Let $\varphi$ be $\partial \varphi$-colinfine. If there exists $t_{0} \in C$ such that $0 \in \partial \varphi\left(t_{0}\right)$, then by the proof of the preceding proposition, $f$ is constant on $C$. Thus, $\left(\mathrm{H}_{0}\right)$ is satisfied and, since $\varphi$ is $\partial \varphi$-colinfine, one has $t^{*}=0$ for any $t^{*} \in \partial \varphi(t)$ and any $t \in C$.

Otherwise, for all $t \in C, t^{*} \in \partial \varphi(t)$, one has $t^{*} \neq 0$. Thus, by Proposition $3.8, \varphi$ is increasing or decreasing on $C$ and then the level set $L_{\varphi}(t)$ of $\varphi$ at $t \in C$ is the singleton $\{t\}$. Now, let $t_{1}^{*}, t_{2}^{*} \in \partial \varphi(t)$ and let $s \in C$, $s>t$. Since $\varphi$ is $\partial \varphi$-colinfine there exist $\lambda_{1}\left(s, t, t_{1}^{*}\right) \in \mathbb{P}$ and $\lambda_{2}\left(s, t, t_{2}^{*}\right) \in \mathbb{P}$ such that

$$
\varphi(s)-\varphi(t)=\lambda_{1}\left(s, t, t_{1}^{*}\right)\left\langle t_{1}^{*}, s-t\right\rangle=\lambda_{2}\left(s, t, t_{2}^{*}\right)\left\langle t_{2}^{*}, s-t\right\rangle .
$$

Thus $t_{1}^{*} t_{2}^{*}>0$.

For the converse, let us first suppose $\left(\mathrm{H}_{0}\right)$ is satisfied and for some $t_{0} \in C$ we have $\partial \varphi\left(t_{0}\right) \ni\{0\}$. Then, for all $s \in C, 0=t_{0}^{*} \in \partial \varphi\left(t_{0}\right)$, we have $\left\langle t_{0}^{*}, s-t_{0}\right\rangle=0$, so that, by $\left(\mathrm{H}_{0}\right), \varphi(s)=\varphi\left(t_{0}\right)$. Hence, $\varphi$ is constant and, in view of conditions $\left(\mathrm{H}^{+}\right)$and $\left(\mathrm{H}^{-}\right)$, for all $r \in C, r^{*} \in \partial \varphi(r)$, we must have $r^{*}=0$ : then $\varphi$ is trivially $\partial \varphi$-colinfine.

Now suppose that for all $t \in C, t^{*} \in \partial \varphi(t)$, the sign of $t^{*}$ is constant on $L_{\varphi}(t)$. Given $r \in C$ such that for all $r^{*} \in \partial \varphi(r), r^{*}>0$, we will prove that $\varphi(t)>\varphi(r)$ for all $t>r$. Let us first prove that we cannot have $\varphi(t)<\varphi(r)$. If $\varphi(t)<\varphi(r)$, let $s:=\sup \{p \in[r, t]: \varphi(p)=\varphi(r)\}$. Since $\varphi$ is continuous, we have $s<t$ and $\varphi(s)=\varphi(r)$. Then our assumption ensures that, for all $s^{*} \in \partial \varphi(s)$, we have $s^{*}>0$,so that, by condition $\left(\mathrm{H}^{+}\right)$ there exists some $p \in] s, t[$ with $\varphi(p)>\varphi(s)$ and the intermediate value theorem yields some $q \in[p, t]$ with $\varphi(q)=\varphi(s)=\varphi(r)$, a contradiction with the definition of $s$. 
Now let us suppose that $\varphi(t)=\varphi(r)$. Then $t^{*}>0$ for any $t^{*} \in \partial \varphi(t)$ by our assumption, so that $\left\langle t^{*}, r-t\right\rangle<0$. Then condition $\left(\mathrm{H}^{-}\right)$yields some $\left.t^{\prime} \in\right] r, t\left[\right.$ such that $\varphi\left(t^{\prime}\right)<\varphi(t)=\varphi(r)$ and replacing $t$ by $t^{\prime}$ in what precedes, we get again a contradiction.

A similar proof shows that $\varphi(q)<\varphi(r)$ for all $q<r$.

We can see in a similar way that when for some $r \in C$, and all $r^{*} \in \partial \varphi(r)$ we have $r^{*}<0$, the function $\varphi$ is decreasing on $C$.

Hence, for any $r, s \in C, s^{*} \in \partial \varphi(s)$ there exists some $\lambda\left(r, s, s^{*}\right) \in \mathbb{P}$ such that

$$
\varphi(r)-\varphi(s)=\lambda\left(r, s, s^{*}\right)\left\langle s^{*}, r-s\right\rangle .
$$

We are ready to prove our main result. It is a nonsmooth version of [5, Theorems 4.13, 4.14], [6], [10], [11], [14].

Theorem 3.1 Let $C$ be an open, convex subset of $X$, let $f: C \rightarrow \mathbb{R}$ be radially continuous and let $\partial f: C \rightrightarrows X^{*}$ with nonempty values.

(a) Let $f$ be $\partial f$-colinfine. Then, for all $w \in C$, the set $K_{w}:=\left\{u \in X: \forall w^{*} \in \partial f(w),\left\langle w^{*}, u\right\rangle=0\right\}$ is a linear subspace of $X$ and $\partial f(w)$ is contained in an half line.

(b) If $f$ is $\partial f$-colinfine on $C$ then for any $w, x \in C$ such that $f(w)=f(x)$ one has $K_{x}=K_{w}$ and for any $w^{*} \in \partial f(w), x^{*} \in \partial f(x)$, there exists some $r>0$ such that $w^{*}=r x^{*}$.

(c) Conversely, suppose that $f$ and $\partial f$ satisfy $\left(H_{0}\right),\left(H^{+}\right)$and $\left(H^{-}\right)$. If for any $w, x \in C$ such that $f(w)=f(x)$ and for any $w^{*} \in \partial f(w), x^{*} \in \partial f(x)$, there exists some $r>0$ such that $w^{*}=r x^{*}$, then $f$ is $\partial f$-colinfine.

(d) Assume that the dimension of $X$ is greater than 1 , that $C=X$, and that $f$ and $\partial f$ satisfy $\left(H_{0}\right),\left(H^{+}\right)$ and $\left(H^{-}\right)$. Then $f$ is $\partial f$-colinfine if, and only if, for all $w, x \in C$ and all $w^{*} \in \partial f(w), x^{*} \in \partial f(x)$ there exists some $r>0$ such that $w^{*}=r x^{*}$.

Proof. (a) It is obvious that $K_{w}$ is a linear subspace. Let $w^{*}, x^{*} \in \partial f(w)$. For any $u \in X$ such that $\left\langle w^{*}, u\right\rangle=0$, taking $t>0$ small enough, we have $w+t u \in C$ and $f(w+t u)=f(w)$. Again, since $f$ is $\partial f$ colinfine, we obtain $\left\langle x^{*}, u\right\rangle=0$. Thus, $\operatorname{ker} w^{*}=K_{w}$ and $\frac{x^{*}}{\left\|x^{*}\right\|}=\frac{w^{*}}{\left\|w^{*}\right\|}$ or $\frac{x^{*}}{\left\|x^{*}\right\|}=-\frac{w^{*}}{\left\|w^{*}\right\|}$ if $w^{*}$ and $x^{*}$ are both non null, while if one is 0 , the other one is 0 too. In other terms, there exists some $r \in \mathbb{R} \backslash\{0\}$ such that $w^{*}=r x^{*}$. Let us prove that $r$ is positive when $x^{*} \neq 0$. Let $y \in C$ be such that $\left\langle x^{*}, y-x\right\rangle \neq 0$. Since $f$ is $\partial f$-colinfine, one has

$$
f(y)-f(x)=\lambda\left(y, x, x^{*}\right)\left\langle x^{*}, y-x\right\rangle=\lambda\left(y, x, w^{*}\right)\left\langle w^{*}, y-x\right\rangle,
$$

hence we get $0 \leq\left\langle x^{*}, y-x\right\rangle\left\langle w^{*}, y-x\right\rangle=\left\langle x^{*}, y-x\right\rangle\left\langle r x^{*}, y-x\right\rangle=r\left\|\left\langle x^{*}, y-x\right\rangle\right\|^{2}$ and $r>0$.

(b) Now let $w, x \in C$ be such that $f(w)=f(x)$ and let $u \in K_{w}$. Since $f$ is $\partial f$-colinfine, we have $x-w \in K_{w}$, hence $x-w+t u \in K_{w}$ for all $t \in \mathbb{R}$ since $K_{w}$ is a linear subspace. Thus, for $|t|$ small enough, we have $x+t u \in C$ and $f(x+t u)=f(w)=f(x)$. Therefore $\left\langle x^{*}, u\right\rangle=0$ for all $x^{*} \in \partial f(x)$ and $u \in K_{x}$. So, $K_{w} \subset K_{x}$. The symmetry of the roles of $w$ and $x$ yields $K_{w}=K_{x}$.

Let $w^{*} \in \partial f(w), x^{*} \in \partial f(x)$. By (a) and the preceding case, we have ker $w^{*}=K_{w}=K_{x}=\operatorname{ker} x^{*}$ and so there exists some $r \in \mathbb{R} \backslash\{0\}$ such that $w^{*}=r x^{*}$. Let us prove that $r$ is positive. Suppose that $r<0$. Pick $u \in X$ such that $\left\langle x^{*}, u\right\rangle=1$ and set $z_{1}=w+t u$ and $z_{2}=x+t u$, with $t>0$ small enough to ensure that $z_{1}, z_{2} \in C$. Then, since $f$ is $\partial f$-colinfine, one has $f\left(z_{1}\right)<f(w)=f(x)<f\left(z_{2}\right)$ and $\left\langle x^{*}, w-x\right\rangle=0$. Since $f$ is radially continuous, there exists some $s \in] 0,1\left[\right.$ such that, for $z:=s z_{1}+(1-s) z_{2}$, one has $f(z)=f(x)$. Then $z-x=s w-s x+t u$ and

$$
0=\left\langle x^{*}, z-x\right\rangle=\left\langle x^{*}, s w-s x+t u\right\rangle=t>0,
$$

a contradiction. Hence, the case $r<0$ is excluded.

(c) If there exist $x \in C$ such that $\partial f(x) \ni 0$, then one has $f(y)=f(x)$ for all $y \in C$ by condition $\left(\mathrm{H}_{0}\right)$ since $\left\langle x^{*}, y-x\right\rangle=0$. Thus, the level set of $f$ at $x$ is $C$ and our assumption ensures that for all $y^{*} \in \partial f(y)$, for some $r>0$, we have $y^{*}=r x^{*}=0$. Thus $f$ is $\partial f$-colinfine. 
Thus, we can assume that $0 \notin \partial f(x)$ for all $x \in C$. Now, given $w, x \in C$, for $t \in C_{w, x}:=\left\{t \in \mathbb{R}: x_{t} \in C\right\}$, where $x_{t}:=(1-t) x+t w$, let us set $\varphi(t):=f\left(x_{t}\right)$. For $t \in C_{w, x}$, let us take

$$
\partial \varphi(t):=\left\{t^{*} \in \mathbb{R}: \exists x_{t}^{*} \in \partial f\left(x_{t}\right), t^{*}=\left\langle x_{t}^{*}, w-x\right\rangle\right\} .
$$

Let us show that $(\varphi, \partial \varphi)$ satisfies $\left(\mathrm{H}^{+}\right),\left(\mathrm{H}^{-}\right)$and $\left(\mathrm{H}_{0}\right)$. Suppose that there exists $t^{*} \in \partial \varphi(t)$ such that $\left\langle t^{*}, s-t\right\rangle>0$ for $t, s \in C_{w, x}$, with $s \neq t$. Then there exists $x_{t}^{*} \in \partial f\left(x_{t}\right)$ such that $\left\langle x_{t}^{*}, x_{s}-x_{t}\right\rangle=(s-t)\left\langle x_{t}^{*}, w-\right.$ $x\rangle=(s-t) t^{*}>0$. Since $f$ satisfies $\left(\mathrm{H}^{+}\right)$, there exists $q$ between $t$ and $s$ such that $\varphi(q):=f\left(x_{q}\right)>f\left(x_{t}\right):=\varphi(t)$. Thus, $\varphi$ satisfies $\left(\mathrm{H}^{+}\right)$. With a similar proof, we can see that $\varphi$ satisfies $\left(\mathrm{H}^{-}\right)$. Now suppose that $\left\langle t^{*}, s-t\right\rangle=0$ for $t, s \in C_{w, x}$, with $s \neq t$ and $t^{*} \in \partial \varphi(t)$. Then there exists $x_{t}^{*} \in \partial f\left(x_{t}\right)$ such that $\left\langle x_{t}^{*}, x_{s}-x_{t}\right\rangle=(s-t) t^{*}=0$. Since $f$ satisfies $\left(\mathrm{H}_{0}\right)$, one has $\varphi(s):=f\left(x_{s}\right)=f\left(x_{t}\right):=\varphi(t)$, hence $\varphi$ satisfies $\left(\mathrm{H}_{0}\right)$.

Suppose that for all $t \in C_{w, x} 0 \notin \partial \varphi(t)$. Let $t, s \in C_{w, x}, t^{*} \in \partial \varphi(t), s^{*} \in \partial \varphi(s)$ be such that $\varphi(t)=$ $\varphi(s)$. We shall prove that $t^{*} s^{*}>0$. Let $x_{t}^{*} \in \partial f\left(x_{t}\right)$ and $x_{s}^{*} \in \partial f\left(x_{s}\right)$ be such that $t^{*}=\left\langle x_{t}^{*}, w-x\right\rangle$ and $s^{*}=\left\langle x_{s}^{*}, w-x\right\rangle$. Since $f\left(x_{t}\right)=\varphi(t)=\varphi(s)=f\left(x_{s}\right)$, there exists some $r>0$ such that $x_{s}^{*}=r x_{t}^{*}$ and we have $s^{*}=\left\langle x_{s}^{*}, w-x\right\rangle=r\left\langle x_{t}^{*}, w-x\right\rangle=r t^{*}$. Hence, we get $t^{*} s^{*}>0$.

Thus, either there exists some $t \in C_{w, x}$ such that $0 \in \partial \varphi(t)$ or for all $t \in C$ the sign of $t^{*} \in \partial \varphi(t)$ is constant on the level set $L_{\varphi}(t):=\varphi^{-1}(\varphi(t))$, hence $\varphi$ is $\partial \varphi$-colinfine, by the preceding lemma.

Moreover, for any $w, x \in C$, for any $x^{*} \in \partial f(x)$, we have $s^{*}:=\left\langle x^{*}, w-x\right\rangle \in \partial \varphi(0)$ and

$$
f(w)-f(x)=\varphi(1)-\varphi(0)=\lambda_{\varphi}\left(1,0, s^{*}\right)\left\langle s^{*}, 1-0\right)=\lambda\left(w, x, x^{*}\right)\left\langle x^{*}, w-x\right\rangle
$$

where $\lambda\left(w, x, x^{*}\right):=\lambda_{\varphi}\left(1,0, s^{*}\right)>0$; thus $f$ is a $\partial f$-colinfine function.

(d) Suppose $C=X$. The sufficiency is clear by (c). Conversely, suppose that $f$ is $\partial f$-colinfine. Given $w, x \in C, w^{*} \in \partial f(w)$ and $x^{*} \in \partial f(x)$ let prove there exists some $r>0$ such that $w^{*}=r x^{*}$.

If $x^{*}=0$, since $f$ is $\partial f$-colinfine, then $f$ is constant and so $w^{*}=0$ : thus the conclusion holds with $r=1$ in this case or in the case $w^{*}=0$. The conclusion also holds when $f(w)=f(x)$, by (b). When $w^{*} \neq 0, x^{*} \neq 0$, $f(w) \neq f(x)$ and there is no $r \in \mathbb{R} \backslash\{0\}$ such that $w^{*}=r x^{*}$ the subspaces $K_{w}$ and $K_{x}$ are the hyperplanes $\operatorname{ker} w^{*}$ and ker $x^{*}$ respectively, so that, the dimension of $X$ being greater than 1, there exists $\bar{z} \in\left(w+K_{w}\right) \cap\left(x+K_{x}\right)$. One has $\bar{z}-w \in K_{w}, \bar{z}-x \in K_{x}$, hence $\left\langle w^{*}, \bar{z}-w\right\rangle=0$ and $\left\langle x^{*}, \bar{z}-x\right\rangle=0$. Since $f$ is $\partial f$-colinfine, we have $f(x)=f(\bar{z})=f(w)$, a contradiction. Then, the existence of some $r>0$ satisfying $w^{*}=r x^{*}$ has been established in (b). Thus this case does not occur. It follows that when $w^{*} \neq 0$ and $x^{*} \neq 0$ there is some $r \in \mathbb{R} \backslash\{0\}$ such that $w^{*}=r x^{*}$. Then we have for some $\lambda, \mu>0, f(x)-f(w)=\lambda\left\langle w^{*}, x-w\right\rangle=\lambda r\left\langle x^{*}, x-w\right\rangle$ and $f(w)-f(x)=\mu\left\langle x^{*}, w-x\right\rangle$, so that $\lambda r=\mu$. Thus $r$ is positive.

Note that [14, Lemma 17, Theorem 18] follow from the preceding lemma and the preceding theorem by taking $\partial f(x):=\{\ell(x)\}$. When $\partial f(x):=\{\nabla f(x)\},[5$, Theorem 4.13,4.14] is a consequence of the preceding theorem.

We devote the rest of this section, to a study of the structure of continuous colinfine functions on a finite dimensional n.v.s. $X$. When $X$ is one-dimensional, it is easy to see that $f$ is quasiaffine if, and only if, it is either nondecreasing or nonincreasing. Thus we suppose that the dimension of $X$ is greater than 1 . The differentiable case is considered in $[4,5,10,11]$ as a $D f$-colinfine function $f$ is pseudoaffine (or pseudolinear in the terminology of these papers). The nonsmooth case is dealt with in [14] with the help of a bifunction. Here, we use a generalized differential $\partial f$ of $f$ and we assume that some natural composition rules are satisfied.

We suppose that when $f=g \circ \ell$ for some non null continuous linear form $\ell$ on $X$ and some continuous function $g: \mathbb{R} \rightarrow \mathbb{R}$, one the following conditions is satisfied:

(C1) $\partial g(\ell(x)) \circ \ell \subseteq \partial(g \circ \ell)(x)$;

$(\mathrm{C} 2) \partial(g \circ \ell)(x) \subseteq \partial g(\ell(x)) \circ \ell$.

Conditions $(\mathrm{C} 1)$ and $(\mathrm{C} 2)$ are satisfied for the Fréchet and the Hadamard subdifferentials.

We deduce from [14], [16], [24] a characterization of continuous $\partial f$-colinfine functions.

Proposition 3.9 Let $f: X \rightarrow \mathbb{R}$ be a lower semicontinuous (resp. continuous), nonconstant function on a finite dimensional n.v.s. If condition (C1) is satisfied, then assertion (a) below implies assertion (b); if condition (C2) is satisfied, the reverse implication holds:

(a) $f$ is $\partial f$-colinfine; 
(b) there exist a continuous linear form $\ell$ on $X, \ell \neq 0$ and a lower semicontinuous (resp. continuous) increasing, $\partial g$-colinfine function $g: \mathbb{R} \rightarrow \mathbb{R}$ such that $f=g \circ \ell$.

Proof. (a) $\Longrightarrow$ (b) Let condition (C1) be satisfy and let $f: X \rightarrow \mathbb{R}$ be l.s.c. (resp. continuous) and $\partial f$ colinfine. Hence $f$ is quasiaffine by Proposition 3.3. According to [16], [24], there exist a continuous linear form $\ell$ on $X$ and a lower semicontinuous. (resp. continuous) nondecreasing function $g: \mathbb{R} \rightarrow \mathbb{R}$ such that $f=g \circ \ell$. Since $f$ is nonconstant, $\ell \neq 0$. Let us prove that $g$ is increasing. Suppose that there exist $r_{1}<r_{2}$ in $\mathbb{R}$ such that for all $r \in\left[r_{1}, r_{2}\right], g(r)=g\left(r_{1}\right)=g\left(r_{2}\right)$. Since $f$ is nonconstant, $g$ is nonconstant and there exists some $t_{0}>1$, such that, for $t \geq t_{0}$ one has either $g\left(r_{1}+t\left(r_{2}-r_{1}\right)\right)>g\left(r_{2}\right)$ or $g\left(r_{2}+t\left(r_{1}-r_{2}\right)\right)<g\left(r_{1}\right)$. We consider the first case, the second one being similar. Since $\ell \neq 0$, there exist $w, x \in X$ such that $\ell(w)=r_{1}<\ell(x)=r_{2}$. Then, for $t \geq t_{0}$, we have $\ell(w+t(x-w))>\ell(x)=r_{2}$ and then $f(w+t(x-w))=g(\ell(w+t(x-w)))=$ $g\left(r_{1}+t\left(r_{2}-r_{1}\right)\right)>g\left(r_{2}\right)=f(w)=f(x)$. By Proposition 3.8, $f$ is not $\partial f$-colinfine. Therefore $g$ is increasing.

Now, we shall prove that $g$ is $\partial g$-colinfine. For any $u, v \in \mathbb{R}$, and $v^{*} \in \partial g(v)$, by condition (C1), there exists $w, x \in X$ and $x^{*} \in \partial f(x)$ such that $\ell(w)=u, \ell(x)=v$ and $v^{*} \circ \ell=x^{*}$. Hence there exists $\lambda_{f}\left(w, x, x^{*}\right) \in \mathbb{P}$ such that

$$
\frac{g(u)-g(v)}{\lambda_{f}\left(w, x, x^{*}\right)}=\frac{g(\ell(w))-g(\ell(x))}{\lambda_{f}\left(w, x, x^{*}\right)}=\left\langle x^{*}, w-x\right\rangle=\left\langle v^{*}, \ell(w)-\ell(x)\right\rangle=\left\langle v^{*}, u-v\right\rangle .
$$

so that $g$ is $\partial g$-colinfine.

(b) $\Rightarrow$ (a) Let condition (C2) be satisfy and $w, x \in X$. Then, for any $x^{*} \in \partial f(x)$, there exists $u^{*} \in \partial g(\ell(x))$ such that $x^{*}=u^{*} \circ \ell$. Since $g$ is $\partial g$-colinfine, there exists $\lambda_{g}\left(\ell(w), \ell(x), u^{*}\right) \in \mathbb{P}$ such that

$$
\frac{f(w)-f(x)}{\lambda_{g}\left(\ell(w), \ell(x), u^{*}\right)}=\frac{g(\ell(w))-g(\ell(x))}{\lambda_{g}\left(\ell(w), \ell(x), u^{*}\right)}=\left\langle u^{*}, \ell(w)-\ell(x)\right\rangle=\left\langle u^{*} \circ \ell, w-x\right\rangle=\left\langle x^{*}, w-x\right\rangle
$$

hence, $f$ is $\partial f$-colinfine.

\section{Characterizations of solution sets}

In the present section, we consider the minimization problem

$$
\min f(x) \text { subject to } x \in C
$$

where $C$ is a subset of n.v.s. $X$ and $f: C \rightarrow \mathbb{R}$.

Our purpose here is limited: since optimality conditions for $(\mathcal{C})$ and mathematical programming problems using the concepts of the previous sections are dealt with in [13], [14], [15], [20], [22], [23], we are just concerned with characterizations of solution sets. Let $S$ be the set of solutions to $(\mathcal{C})$ and let $\partial f: C \rightrightarrows X^{*}$ be a generalized differential of $f$.

The following results are direct consequences of Definition 2.3. Here we set

$$
S_{a}:=\left\{x \in C: \exists a^{*} \in \partial f(a),\left\langle a^{*}, x-a\right\rangle=0\right\}, \quad S_{a}^{\prime}:=\left\{x \in C: \forall a^{*} \in \partial f(a),\left\langle a^{*}, x-a\right\rangle=0\right\} .
$$

Proposition 4.1 Let $f$ be $\partial f$-colinfine on $C$.

(a) If $a \in S$, then for all $x \in C, a^{*} \in \partial f(a), x^{*} \in \partial f(x)$ one has $\left\langle a^{*}, x-a\right\rangle \geq 0$ and $\left\langle x^{*}, a-x\right\rangle \leq 0$.

Moreover, $S=S_{a}=S_{a}^{\prime}$.

(b) Conversely, if for all $x \in C$, either there exists $a^{*} \in \partial f(a)$ such that $\left\langle a^{*}, x-a\right\rangle \geq 0$ or there exists $x^{*} \in \partial f(x)$ such that $\left\langle x^{*}, a-x\right\rangle \leq 0$, then $a \in S$.

Proposition 4.2 Let $f$ be an eventually $\partial f$-colinfine function on $C$.

(a) If $a \in S$, then for all $x \in C$, there exists some $a^{*} \in \partial f(a)$ such that $\left\langle a^{*}, x-a\right\rangle \geq 0$ and there exists some $x^{*} \in \partial f(x)$ such that $\left\langle x^{*}, a-x\right\rangle \leq 0$.

Moreover, $S_{a}^{\prime} \subset S \subset S_{a}$.

(b) Conversely, if for all $x \in C$, either for all $a^{*} \in \partial f(a)$ one has $\left\langle a^{*}, x-a\right\rangle \geq 0$ or for all $x^{*} \in \partial f(x)$ one has $\left\langle x^{*}, a-x\right\rangle \leq 0$ then $a \in S$. 
Let us consider now the case in which the constraint set $C$ is defined by a finite family of inequalities, so that problem $(\mathcal{C})$ turns into the mathematical programming problem

$$
(\mathcal{M}) \min f(x) \text { subject to } x \in C:=\left\{x \in X: g_{1}(x) \leq 0, \ldots, g_{n}(x) \leq 0\right\},
$$

where $f: W \rightarrow \mathbb{R}, g_{i}: W \rightarrow \mathbb{R}$ and $W$ is a subset of $X$. Let $a \in C$, let $I:=\{1, \ldots, n\}$ and let $I(a):=\{i \in I$ : $\left.g_{i}(a)=0\right\}$.

In convex mathematical programming, the multipliers are the same for all solutions. The following result follows from [14]. Note here instead of colinvexity of the objective function $f$, we only need that $f$ is $\partial f$ protoconvex.

Proposition 4.3 Let $a \in S$ be such that there exists $y=\left(y_{i}\right) \in \mathbb{R}_{+}^{n}, a^{*} \in \partial f(a), a_{i}^{*} \in \partial g_{i}(a)$ for $i \in I(a)$ satisfying $a^{*}+\sum_{i \in I(a)} y_{i} a_{i}^{*}=0$ and $y_{i} g_{i}(a)=0$ for $i \in I$.

(a) If either $f$ is $\partial f$-colinfine at a or $f$ is $\partial f$-protoconvex at $a$ and $g_{i}$ is $\partial g_{i}$-protoconvex at $a(i \in I(a))$, then $\sum_{i \in I(a)} y_{i}\left\langle a_{i}^{*}, b-a\right\rangle=0$ for every $b \in S$.

(b) Let $f$ be $\partial f$-protoconvex at $a$ and $g_{i}$ be $\partial g_{i}$-colinvex at $a(i \in I(a))$. Let $L$ be the Lagrangian given by $L(x, u):=f(x)+u g(x)$ for $(x, u) \in C \times \mathbb{R}_{+}^{n}$ and let $b \in C$. Then, $b \in S$ if, and only if, $y g(b)=0$ and $L(a, y)=L(b, y)$.

Proof. (a) Let $a^{*} \in \partial f(a), a_{i}^{*} \in \partial g_{i}(a)$ for $i \in I(a), y \in \mathbb{R}_{+}^{n}$ and $b \in S$ be as in the statement.

If $f$ is $\partial f$-colinfine at $a$, then for $\mu:=\lambda\left(b, a, a^{*}\right)>0$ one has for $b \in S$

$$
0=\left\langle a^{*}, b-a\right\rangle+\left\langle\sum_{i \in I(a)} y_{i} a_{i}^{*}, b-a\right\rangle=\frac{f(b)-f(a)}{\mu}+\left\langle\sum_{i \in I(a)} y_{i} a_{i}^{*}, b-a\right\rangle=\left\langle\sum_{i \in I(a)} y_{i} a_{i}^{*}, b-a\right\rangle .
$$

Now, assume that $f$ is $\partial f$-protoconvex at $a$ and $g_{i}$ is $\partial g_{i}$-protoconvex at $a(i \in I(a))$. Since $f(b)=f(a)$, one has $\left\langle a^{*}, b-a\right\rangle \leq 0$. Thus, $\sum_{i \in I(a)} y_{i}\left\langle a_{i}^{*}, b-a\right\rangle \geq 0$. On the other hand, since $g_{i}(b) \leq g_{i}(a)=0$ for $i \in I(a)$, one has $\left\langle a_{i}^{*}, b-a\right\rangle \leq 0$. Thus, we get $\sum_{i \in I(a)} y_{i}\left\langle a_{i}^{*}, b-a\right\rangle=0$.

(b) If $g_{i}$ is $\partial g_{i}$-colinvex at $a(i \in I(a))$, then there exist $\lambda_{i}>0$ such that

$$
0 \geq g_{i}(b)=g_{i}(b)-g_{i}(a) \geq \lambda_{i}\left\langle a_{i}^{*}, b-a\right\rangle .
$$

Set $\lambda:=\max \left\{\lambda_{i}: i \in I(a)\right\}$. Then, for all $i \in I(a)$ one has $g_{i}(b) \geq \lambda\left\langle a_{i}^{*}, b-a\right\rangle$.

By (a), $y \in \mathbb{R}_{+}^{n}$ and $b \in C$, one gets $0 \geq y g(b) \geq \lambda \sum_{i \in I(a)} y_{i}\left\langle a_{i}^{*}, b-a\right\rangle=0$. Moreover, we have $L(a, y)=L(b, y)$ since $f(a)+y g(a)=f(a)=f(b)=f(b)+y g(b)$.

Now for the converse, let $b \in C$ be such that $y g(b)=0$ and $L(a, y)=L(b, y)$. Then, we have $f(a)=$ $L(a, y)=L(b, y)=f(b)+y g(b)=f(b)$, hence $b \in S$.

\section{References}

[1] Aggarwal, S., and Bhatia, S., Pseudolinearity and efficiency via Dini derivatives. Indian Journal of Pure and Applied Mathematics, Vol. 20, No. 12, pp. 1173-1183, 1989.

[2] Aussel, D., and Hadjisavvas, N., On quasivariational inequalities, Journal of Optimization Theory and Applications, Vol. 121, No. 2, pp. 445-450, 2004.

[3] Bianchi, M., and Schaible, S., An extension of pseudolinear functions and variational inequalities problems, Journal of Optimization Theory and Applications, Vol. 104, No. 1, pp. 59-71, 2000.

[4] Cambini, R., and CArosi, L. On generalized linearity of quadratic fractional functions, Journal of Global Optimization, Vol. 30, No. 2-3, pp. 235-251, 2004. 
[5] Cambini, A., and Martein, L., Generalized convexity and optimality conditions in scalar and vector optimization, In: Handbook of generalized convexity and generalized monotonicity, N. Hadjisavvas, S. Komlosi and S. Schaible (eds.), Kluwer Academic Publishers, pp. 151-194, 2005.

[6] Chew, K. L., and Choo, E. V., Pseudolinearity and efficiency, Mathematical Programming, Vol. 28, pp. 226-239, 1984.

[7] Demyanov, V. F., and Rubinov, A. M., Contructive nonsmooth analysis, Verlag Peter Lang, Frankfurt am Main, 1995.

[8] JEYAKuma, V., and LuC, D. T., Nonsmooth vector functions and continuous optimization, Springer (book to appear).

[9] Jeyakuma, V., and YAnG, X. Q., On characterizing the solution sets of pseudolinear programs, Journal of Optimization Theory and Applications, Vol. 87, No. 3, pp. 747-755, 1995.

[10] Komlosi, S., First and second characterizations of pseudolinear functions, European Journal of Operation Research, Vol. 67, pp. 278-286, 1993.

[11] Kortanek, K. O., and Evans, J. P., Pseudoconcave programming and Lagrange regularity, Oper. Res., Vol. 67, pp. 278-286, 1993.

[12] Kruk, S., and Wolkowicz, H., Pseudolinear programming, SiAM Review. Vol. 41, No. 4, pp. 795-805, 1999.

[13] Linh, N. T. H., and PENOT, J.-P., Optimality conditions for quasiconvex programming, SIAM Journal Optimization, Vol. 17, No. 2, pp. 500-510, 2006.

[14] Linh, N. T. H., and PEnOt, J.-P., Generalized affine maps and generalized convex functions, preprint, University of Pau, July 2006.

[15] Linh, N. T. H., and PEnOt, J.-P., Generalized convex functions and generalized differentials, preprint, University of Pau, October 2006.

[16] Martinez-LeGAZ, J. E., Quasiconvex duality theory by generalized conjugation methods, Optimization, Vol. 19, pp. 603-652, 1988.

[17] Martos, B., Nonlinear programming: Theory and methods, North-Holland, Amsterdam, Holland, 1975.

[18] Rapcsak, T., On pseudolinear functions, European Journal of Opererational Research. Vol. 50, No. 3, pp. 353-360, 1991.

[19] SACH, P.H., and PENOT, J.-P., Characterizations of generalized convexities via generalized directional derivatives. Numerical Functional Analysis and Optimization, Vol. 19, pp. 615-634, 1998.

[20] Penot, J.-P., Are generalized derivatives useful for generalized convex function. In: Generalized Convexity, Generalized Monotonicity, J.-P.Crouzeix, M. Volle, J.-E. Martinez-Legaz (eds.), Kluwer Academic Publishers, pp. 3-39, 1998.

[21] Penot, J.-P., What is quasiconvex analysis?, Optimization, Vol. 47, pp.35-110, 2000.

[22] PEnOt, J.-P., Characterization of solution sets of quasiconvex programs, Journal of Optimization Theory and Applications, Vol. 117, No. 3, pp. 627-636, 2003.

[23] Penot, J.-P., A Lagrangian approach to quasiconvex analysis, Journal of Optimization Theory and Applications, Vol. 117, No. 3, pp. 637-647, 2003. 
[24] PEnOt, J.-P., Glimpses upon quasiconvex analysis, ESIAM: Proceedings, to appear.

[25] Penot, J.-P., and SACH, P. H., Generalized monotonicity of subdifferentials and generalized convexity, Journal of Optimization Theory and Applications, Vol. 94, No. 1, pp. 251-262, 1997.

[26] Penot, J.-P., and QUAng, P. H., Generalized convexity of functions and generalized monotonicity of set-valued maps, Journal of Optimization Theory and Applications, Vol. 92, No. 2, pp. 343-356, 1997. 